\title{
Conduction in ulnar nerve bundles that innervate the proximal and distal muscles: a clinical trial
}

\author{
Attila Oğuzhanoğlu*, Sibel Güler ${ }^{2}$, Mustafa Çam ${ }^{3}$, Eylem Değirmenci ${ }^{1}$
}

\begin{abstract}
Background: This study aims to investigate and compare the conduction parameters of nerve bundles in the ulnar nerve that innervates the forearm muscles and hand muscles; routine electromyography study merely evaluates the nerve segment of distal (hand) muscles.

Methods: An electrophysiological evaluation, consisting of velocities, amplitudes, and durations of ulnar nerve bundles to 2 forearm muscles and the hypothenar muscles was performed on the same humeral segment.

Results: The velocities and durations of the compound muscle action potential (CMAP) of the ulnar nerve bundle to the proximal muscles were greater than to distal muscles, but the amplitudes were smaller.

Conclusions: Bundles in the ulnar nerve of proximal muscles have larger neuronal bodies and thicker nerve fibers than those in the same nerve in distal muscles, and their conduction velocities are higher. The CMAPs of proximal muscles also have smaller amplitudes and greater durations. These findings can be attributed to the desynchronization that is caused by a wider range of distribution in nerve fiber diameters.

Conduction parameters of nerve fibers with different diameters in the same peripheral nerve can be estimated.
\end{abstract}

\section{Background}

Peripheral motor nerve diameter decreases gradually after emerging from the spinal cord toward the target muscles. In a myelinated nerve fiber, the thickness of the fiber correlates positively with nerve conduction velocity; conduction velocity declines when a fiber's diameter decreases[1].

Nerve diameter is proportional to the size of the motor nerve body in the anterior horn [1]. Nerve diameter thickness and conduction velocity correlate with nerve body size. Proximal muscles with bigger masses are innervated by thicker fibers [2-5].

In addition to the sciatic nerve $[4,6]$, the nerve velocities in the ulnar nerve can be recorded and calculated separately between the proximal and distal muscles of the upper extremities. Thus, one can differentiate between the fastest conductive fibers that innervate the proximal and distal muscles electrophysiologically.

In this study, we examined the nerve conduction velocities, compound muscle action potential (CMAP)

\footnotetext{
* Correspondence: atofirst@gmail.com

'Pamukkale University, School of Medicine, Department of Neurology,

Araştırma Hastanesi, Kınıkı-Denizli, Turkey

Full list of author information is available at the end of the article
}

amplitudes, and duration of 2 proximally positioned forearm muscles that have greater mass and hypothenar muscles that are distally positioned with smaller mass.

\section{Methods}

This study was performed using cases that were referred for evaluation in the EMG laboratory (Premiere Plus EMG Device. Medelec/Vickers Medical, Manor Way, Old Woking, Surrey, United Kingdom, GU22 9JU) and was approved by the Local Ethical Committee (Clinical Researches Committee, Denizli Province, Ministry of Health, Republic of Turkey). All patients gave informed consent. The patients were included after undergoing a routine electrophysiological protocol to exclude polyneuropathy and any neuropathy. The study was performed in the right upper extremity in all normative subjects (30 subjects: 6 men, 24 women). The mean $( \pm \mathrm{SD})$ age of the patients was $38.7( \pm 15.4)$ (Range 16-70). Room temperature always exceeded $24^{\circ} \mathrm{C}$. The subject was lain down. The right arm was positioned 70-90 degrees to the body, and the forearm lay 90 degrees to the arm; this position was maintained throughout the study. 


\section{Stimulus}

1. Ulnar nerve stimuli in the arm segment: A point, $5 \mathrm{~cm}$ proximal to the medial epicondyle, was selected as the distal stimulus point. A point, $12 \mathrm{~cm}$ proximal to the distal point in the axillary region, was the proximal stimulus point.

2. Ulnar nerve stimuli in the forearm segment: A point, $5 \mathrm{~cm}$ distal to the medial epicondyle, was chosen as the proximal stimulus point. A point, $5 \mathrm{~cm}$ proximal to the distal wrist line, served as the distal stimulus point.

The supramaximal level of the stimulus intensity was increased slowly until a point was reached at which the CMAP amplitude no longer increased. The point at which it rose $25 \%$ more to ensure that the amplitude did not change further was selected as the severity of stimulus in all cases, and the stimulus duration was $100 \mu \mathrm{s}$.

\section{Recording}

\section{1 - Forearm recording}

A-Recording from the flexor carpi ulnaris muscle (FCU): The active disc electrode (silver, $10 \mathrm{~mm}$ in diameter) was positioned at a point 2 digits wide from the ulna, where the proximal third and medial third sections of the forearm met. The reference electrode was positioned on the ulna, transverse to the active electrode $[7,8]$.

B - Recording from the flexor digitorum profundus muscle (FDP): The recording was made by a bar electrode $(40 \mathrm{~mm}$ in length, $20 \times 8 \mathrm{~mm}$ recording surface for both the anode and cathode); the active part was positioned on the medial third of the forearm, near the ulna, and the reference was positioned distally [7].

2 - Hand (Hypothenar-HYT, abductor digiti minimi muscleADM) recording

The active bar electrode was positioned at the midpoint between the wrist distal line and the metacarpophalangeal joint [8]. The reference bar electrode was positioned on the metacarpophalangeal joint, a more distal position.

The screen sweep time was $30 \mathrm{~ms}$, and the sensitivity was $2-5 \mathrm{mV}$.

\section{Study scheme}

After the subject lay down and the right upper extremity was positioned as discussed, the FCU, FDP, and HYT muscles were recorded alternatively by stimulating the arm segment separately for each recording. Then, a recording of the HYT muscle was made by stimulating the forearm. Thus, nerve conduction velocities and CMAP amplitude and duration were measured in the 3 responses from 3 different muscles in the arm and hand. Amplitude was measured from onset to the negative peak, and duration was measured from the onset of the first negative deflection to the last point at which the potential returned to baseline. Latency was measured from the stimulus artefact to the onset of negative deflection. Additionally, nerve conduction velocity and CMAP amplitude and duration in the forearm segment was measured in the responses from the ADM (HYT) muscle.

\section{Statistics}

Kolmogorov-Smirnov test was performed for the appropriateness of data distribution to the normal distribution. Because all data were distributed normally, we used t-test in paired groups and Bonferroni correction for data comparisons and Pearson's correlation analysis to determine the correlation between the data. $\mathrm{p}<.05$ was the significance level. SPSS v.16 was used for all statistical evaluations.

\section{Results}

Ulnar nerve conduction velocities

Velocities from 2 forearm muscles (FCU, FDP) and 1 hand muscle (HYP) after stimulation of the arm segment were compared. These velocities were also compared with that obtained of the HYP area after stimulation of the forearm segment. The comparison of these velocities are shown in Table 1.

The velocities $V_{F C U}$ and $V_{F D P}$ in the arm segment did not differ, as calculated by the responses from both forearm muscles.

The velocities in the hypothenar area did not differ between the arm segment $\left(\mathrm{V}_{\mathrm{HYP}}\right)$ and forearm segment ( $\left.\mathrm{V}_{\text {HYP-FA }}\right)$.

Both velocities of the arm segment $\left(\mathrm{V}_{\mathrm{FCU}}, \mathrm{V}_{\mathrm{FDP}}\right)$ from the forearm muscles exceeded those from the arm segment $\left(\mathrm{V}_{\mathrm{HYP}}\right)$ and forearm segment $\left(\mathrm{V}_{\mathrm{HYP}-\mathrm{FA}}\right)$, both of which were recorded from the hypothenar area.

Table 1 Nerve conduction velocities after stimulus on the arm and forearm segments

\begin{tabular}{|c|c|c|c|c|c|}
\hline Velocity & Mean $( \pm S D)(m / s n)$ & $\mathbf{n}$ & Comparison & $\mathrm{T}$ value & $\mathbf{P}$ \\
\hline$V_{F C U}$ & 76.27 (12.67) & 30 & $V_{F C U}-V_{F D P}$ & -0.41 & 0.685 \\
\hline$V_{\text {FDP }}$ & 76.25 (13.36) & 30 & $V_{F C U}-V_{H Y P}$ & 5.61 & 0.000 \\
\hline$V_{\text {HYP }}$ & $61.90(7.21)$ & 30 & $V_{F C U}-V_{\text {HYP-FA }}$ & 6.32 & 0.000 \\
\hline \multirow[t]{3}{*}{$V_{\text {HYP-FA }}$} & $62.26(5.05)$ & 30 & $V_{F D P}-V_{H Y P}$ & 5.87 & 0.000 \\
\hline & & & $V_{F D P}-V_{\text {HYP-FA }}$ & 5.44 & 0.000 \\
\hline & & & $V_{\text {HYP }}-V_{\text {HYP-FA }}$ & -0.26 & 0.793 \\
\hline
\end{tabular}

m: meter, s: second

$V_{\mathrm{FCU}}$ : Ulnar nerve conduction velocity in the FCU muscle

$V_{F D P}$ : Ulnar nerve conduction velocity in the FDP muscle

$\mathrm{V}_{\mathrm{HYP}}$ : Ulnar nerve conduction velocity in the HYP area muscle after stimuli on the arm segment

$\mathrm{V}_{\text {HYP-FA }}$ : Ulnar nerve conduction velocity in HYP area muscles after stimuli on the forearm segment 


\section{Compound muscle action potentials A-Amplitude}

In the CMAP evaluation, comparisons were made by calculating the average CMAP amplitudes, obtained after proximal and distal stimulation of each muscle. CMAP amplitudes of 2 forearm muscles $\left(\mathrm{AMP}_{\mathrm{FCU}}\right.$, $\left.\mathrm{AMP}_{\mathrm{FDP}}\right)$ and 1 hand muscle $\left(\mathrm{AMP}_{\mathrm{HYP}}\right)$ after stimulation of the arm segment were compared with the amplitude $\left(\mathrm{AMP}_{\mathrm{HYP}-\mathrm{FA}}\right)$ from the HYP area after stimulation of the forearm segment. These comparisons are shown in Table 2.

The CMAP amplitudes of the forearm muscles $\left(\mathrm{AMP}_{\mathrm{FCU}}, \mathrm{AMP}_{\mathrm{FDP}}\right)$ did not differ.

The CMAP amplitudes from both forearm muscles $\left(\mathrm{AMP}_{\mathrm{FCU}}, \mathrm{AMP}_{\mathrm{FDP}}\right)$ were lower than the 2 amplitudes of the hypothenar $\left(\mathrm{AMP}_{\mathrm{HYP}}\right.$ and $\left.\mathrm{AMP}_{\mathrm{HYP}-\mathrm{FA}}\right)$.

The amplitude in the hypothenar area $\left(\mathrm{AMP}_{\mathrm{HYP}}\right)$ was lower compared with that of the forearm $\left(\mathrm{AMP}_{\mathrm{HYP}-\mathrm{FA}}\right)$ segment.

\section{$B$ - Response duration}

We compared the average CMAP duration after proximal and distal stimulation. CMAP durations from the 2 forearm muscles (DUR $\mathrm{FCU}, \mathrm{DUR}_{\mathrm{FDP}}$ ) and 1 hand muscle (DUR $\mathrm{HYP}_{\mathrm{HP}}$ ) after stimulation of the arm segment were compared with that from the HYP area after stimulation of the forearm segment. This comparison is shown in Table 3.

We observed no difference in CMAP duration between the forearm muscles (DUR $\left.\mathrm{FCU}_{\mathrm{FU}}, \mathrm{DUR}_{\mathrm{FDP}}\right)$.

CMAP duration in the hypothenar area $\left(\mathrm{DUR}_{\mathrm{HYP}}\right)$ and forearm (DUR $\left.\mathrm{BYP}_{\mathrm{H}-\mathrm{FA}}\right)$ did not differ.

The CMAP duration in both forearm muscles $\left(D_{U} R_{F C U}, D R_{F D P}\right)$ was longer than that in the hypothenar areas (DUR HYP and DUR HYP-FA $_{\text {. }}$.

\section{Correlation between average velocity, amplitude, and} duration

The correlation between the 4 groups of velocities, amplitudes, and durations is shown in Table 4. A strong

Table 2 CMAP amplitudes after stimulus on the arm and forearm segments

\begin{tabular}{|c|c|c|c|c|c|}
\hline Amplitude & $\begin{array}{l}\text { Mean }( \pm S D) \\
(\mathrm{mV})\end{array}$ & $n$ & Comparison & $\begin{array}{l}\text { T } \\
\text { value }\end{array}$ & $\mathbf{P}$ \\
\hline $\mathrm{AMP}_{\mathrm{FCU}}$ & $3.28(1.33)$ & 30 & $A M P_{F C U}-A M P_{F D P}$ & -0.43 & 0.670 \\
\hline$A M P_{F D P}$ & $3.17(1.48)$ & 30 & $A M P_{F C U}-A M P_{H Y P}$ & -6.83 & 0.000 \\
\hline $\mathrm{AMP}_{\mathrm{HYP}}$ & $5.87(1.81)$ & 30 & $A M P_{F C U}-A M P_{H Y P-F A}$ & -8.51 & 0.000 \\
\hline \multirow[t]{3}{*}{$\mathrm{AMP}_{\mathrm{HYP}-\mathrm{FA}}$} & $6.47(2.06)$ & 30 & $A M P_{F D P}-A M P_{H Y P}$ & -7.38 & 0.000 \\
\hline & & & $A M P_{F D P}-A M P_{H Y P-F A}$ & -8.89 & 0.000 \\
\hline & & & $A M P_{H Y P}-A M P_{H Y P-F A}$ & -3.55 & 0.001 \\
\hline
\end{tabular}

mV:millivolt

$\mathrm{AMP}_{\mathrm{FCU}}$ : CMAP amplitude in the FCU muscle

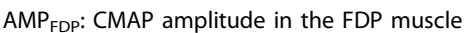

$\mathrm{AMP}_{\text {HYP }}$ : CMAP amplitude in the HYP area muscle after stimulus on the arm segment

AMP $_{\text {HYP-FA: }}$ CMAP amplitude in the HYP area muscles after stimulus on the forearm segment
Table 3 CMAP duration after stimulus on the arm and forearm segments

\begin{tabular}{|c|c|c|c|c|c|}
\hline Duration & Mean $( \pm \mathrm{SD})(\mathrm{ms})$ & $\mathrm{n}$ & Comparison & $T$ value & $P$ \\
\hline $\mathrm{DUR}_{\mathrm{FCU}}$ & $7.45(1.26)$ & 30 & $D U R_{F C U}-D U R_{F D P}$ & -1.87 & 0.071 \\
\hline DUR & $7.97(1.37)$ & 30 & DURFCU - DUR & -5.53 & 0.000 \\
\hline DUR $_{H Y P}$ & $5.90(1.04)$ & 30 & DURFCU $-D U R_{H Y P-F A}$ & 7.44 & 0.000 \\
\hline \multirow[t]{3}{*}{ DUR $R_{H Y P-F A}$} & $5.76(0.88)$ & 30 & $D U R_{F D P}-D U R_{H Y P}$ & -7.38 & 0.000 \\
\hline & & & $D U R_{F D P}-D U R_{H Y P-F A}$ & 7.96 & 0.000 \\
\hline & & & DUR $R_{H Y P}-D U R_{H Y P-F A}$ & 0.68 & 0.504 \\
\hline
\end{tabular}

ms: millisecond

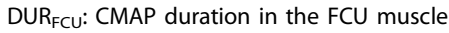

DUR $_{F D P}$ : CMAP duration in the FDP muscle

DUR $_{\text {HYP: }}$ CMAP duration in the HYP area muscle after stimulus on the arm segment

DUR $_{\text {HYP-FA: }}$ CMAP duration in the HYP area muscles after stimulus on the forearm segment

negative correlation from arm to forearm changes was observed between velocity and amplitude and between amplitude and duration. In addition, velocity and duration had a strong positive correlation.

\section{Discussion}

In studies in the hind limb of rats [4] and lower extremities in humans [6], n. tibialis fibers that extend to the $\mathrm{m}$. gastrocnemius, which is a proximal muscle, conduct faster than the tibial nerve fibers that connect to interosseous muscles, the small muscles of the feet.

Cullheim [1] has shown that motor neuron size in the anterior horn correlates positively with intramedullary axon diameter and axon conduction velocity and that the correlation between the first axon segment and axon conduction velocity is the most powerful one.

In their study on the hind limb in mouse, McHanwell and Biscoe [3] examined motor neuron body areas and showed that body areas of the nerves that travel to proximally positioned femoral muscles are larger than those of distally positioned crucial muscles. The histograms of body area of proximally positioned muscles are bimodal, and those of the distal foot muscles are unimodal.

Fernand and Young [9] demonstrated that nerves of proximally positioned muscles are thicker than those of distally positioned muscles in the upper and lower extremities in rabbit. Histograms of the diameters of proximal muscles nerves show a bimodal distribution,

Table 4 Correlations between velocity, amplitude, and duration*

\begin{tabular}{lll}
\hline Variables & $\mathbf{r}$ & $\mathbf{P}$ \\
\hline Velocity-Amplitude & -0.987 & 0.013 \\
Velocity-Duration & 0.986 & 0.014 \\
Amplitude-Duration & -0.982 & 0.018 \\
\hline
\end{tabular}

*: Pearson's correlation analysis. r:correlation coefficient. 
while those of distal foot interosseous muscles are unimodal. They noted in their classification of nerve fibers that a considerable percentage of bimodally distributed nerve fibers exceeded $14 \mu \mathrm{m}$ in diameter and can reach $24 \mu \mathrm{m}$ and that a minute proportion of unimodal muscular fibers are 10-12 $\mu \mathrm{m}$; most of them, however, fall below these values.

Devanandan et al. [10] noted a similarity and unimodal distribution between histograms of the profound branch of the n. ulnaris in Bonnet monkeys (Macaca radiata) and the profound branch of the human n. ulnaris. Most hand muscles (Flexor digiti minimi, opponens digiti minimi, adductor pollicis, and the first dorsal interosseal muscles) showed unimodal distribution; the abductor digiti minimi showed bimodal distribution. Furthermore, the authors emphasized that none of the nerve fibers in these muscles exceeded $12 \mu \mathrm{m}$.

Buchtal and Schmalbruch [2] has suggested that with regard to motor unit size, conduction velocity changes according to the size of the motor neuron and muscle mass; because proximally positioned muscles are larger in mass, they are believed to have larger motor neurons.

This is the first study that compares conduction velocities in fibers that enter the ulnar nerve and reach 2 disparate muscle groups in the arm and hand segments. It has been shown electrophysiologically that nerve fibers that innervate 2 muscles that are proximally positioned and larger in mass (FCU and FDP) conduct faster than those that innervate distal muscles that are smaller. This finding indicates that nerve fibers of the proximal muscles are thicker in the arm segment.

Nerves branch and become thinner conically after emerging from the spinal cord during their march toward the muscles, becoming even thinner [9]. Because the conduction velocity of the proximally and distally positioned muscles was measured in the same arm segment in this study, we propose that the difference between the velocities does not depend on proximodistal thinning of the nerve diameter. Based on our results, conduction velocities are higher in fibers of the ulnar nerve of proximal muscles (FCU, FDP), which have larger neuronal bodies and thicker nerve fibers than fibers in the same nerve of the distal muscles (HYP).

No difference was observed between the conduction velocities in the arm and forearm segments by hypothenar recording, but data are conflicting on this subject. The chief problem is whether the velocity in the elbow segment is included in the proximal or distal segment velocity. Harding and Halar [11] have opined how ulnar nerve conduction is influenced by elbow angle in humans and cadavers, demonstrating that conduction time increases in the forearm and that motor conduction velocity decreases in the forearm segment if the forearm is positioned $45^{\circ}$ from the flexion position to extension. They have reported in cadavers that increasing elbow flexion decreases ulnar nerve wrinkledness and that the nerve relocates distally and becomes smoother in the above-elbow segment [11].

Flexion of the forearm affects not only the position of the ulnar nerve in the elbow segment but also the position of the nerve in the arm and forearm segments by shifting the nerve in these segments. It has been suggested that flexion in the elbow beyond $90^{\circ}$ does not increase conduction velocity, and recordings in the hypothenar area with the elbow in this position have shown that the velocities in the 3 segments are equal and that $90^{\circ}$ flexion is the most suitable position [12]. In our study, with the elbow in $90^{\circ}$ flexion, there was no difference between conduction velocities in the arm and forearm segments in the hypothenar recordings, demonstrating that diameter thinning in the nerve fibers that innervate the hypothenar muscles precludes them from reaching to an extent that is sufficient to cause changes in conduction velocity.

In proportion to the distance between the stimulus point and recording point, the sensorial (and also less marked in motor) action potential amplitudes and areas decrease and the response duration climbs[13]. This finding is due temporal dispersion [13-15]. In our study, arm segment amplitudes in the HYT recording were smaller than the forearm segment amplitudes in the HYT recording, and there was no difference between the durations.

Notably, the amplitudes from the forearm muscles (FCU, FDP) were smaller than those in the HYP area, and the durations were longer than the HYP response durations. Differences in temperature have been proposed to explain the disparities between the arm and forearm segments in nerve conduction studies. Although the effect of temperature on nerve conduction velocity has found near-total acceptance, its effect on amplitude has not. Studies have measured temperature differences between arm and forearm segments of $0.6^{\circ} \mathrm{C}$ [16] and $1.1^{\circ} \mathrm{C}$ [17]. Both have stated that there is no relation between variations in temperature and conduction velocity[16,17]. Todnem et al. [18] have suggested that the median nerve motor amplitude does not undergo significant alterations with temperature changes.

We need to find factors other than temperature that explain the differences in amplitude between proximal and distal muscles in this study. Fernand and Young [9] reported that nerve diameters of proximal muscles show bimodal distribution in rabbits, exceeding those of nerves in distal muscles, which had unimodally distributed nerve fibers. They also found that nerve fibers of muscles with unimodal distribution had primarily the same diameter, failing to observe nerve fibers that had large or tiny diameters. There are fibers that have very 
small and very large diameters, and nerves in proximal forearm muscles have a wide range of diameters. Based on the results of Devanandan et al.[10] and our study, we postulate that nerve fibers of the FCU and FDP muscles are bimodal and that nerve fibers of HYP area muscles are unimodally distributed, assuming that similar features exist in humans.

Proximal muscles should have nerve fibers with a wide range of diameters, and distal muscles should have a narrower range of nerve fiber diameters. For example, nerve fibers in the $\mathrm{m}$. semimembranosus, a proximal muscle in the posterior extremity in rabbit, have diameters that shift between $2-20 \mu \mathrm{m}$, and those of nerve fibers in the $\mathrm{m}$. interosseous, a distal muscle, are between 2-12 $\mu \mathrm{m}$ [9]. In our study, the reduction in CMAP amplitude and prolongation in response duration in the forearm proximal muscles might be caused by the desynchronization that arises from the magnitude of the temporal dispersion that is caused by differences between conduction velocities of their nerve fibers. This situation is comparable with the desynchronization that is caused increased stimulus distances - the result of temporal dispersion during recording in the HYP region $[14,15]$.

With regard to the relationship between parameters of nerve conduction velocities, velocity and response duration correlated positively, and a negative correlation was observed between velocity and amplitude and between amplitude and duration, all of which were strong. Nerve conduction velocity decreased from the proximal to the distal, as did duration, and amplitude increased (Table 4).

\section{Conclusion}

Nerve bundles that travel to proximally positioned muscles (FCU, FDP) that are innervated by the ulnar nerve have higher nerve conduction velocities compared with those of bundles that extend to distally positioned muscles (HYP). The CMAP amplitudes of proximal muscles are lower than those of distal muscles, and their duration is longer. This finding is attributed to the desynchronization that is caused by the wide range in diameters of nerve fibers in the proximal muscles, arising during nerve stimulation and after temporal dispersion. Although our sample size was not high, we believe that separate EMG recordings from proximal and distal muscles might yield insights into nerve fibers and size of motor nerve cell bodies.

\section{Abbreviations}

EMG: Electromyography; CMAP: compound muscle action potential; FCU: flexor carpi ulnaris muscle; FDP: flexor digitorum profundus muscle; HYT: Hypotenar area; ADM: abductor digiti minimi muscle; AMPFCU: CMAP amplitude in the FCU muscle; AMP FDP: CMAP amplitude in the FDP muscle;
AMP $_{\text {HYP: }}$ CMAP amplitude in the HYP area muscle after stimulus on the arm segment; AMP HYP-FA: CMAP amplitude in the HYP area muscles after stimulus on the forearm segment; DUR $F C U$ : CMAP duration in the FCU muscle;

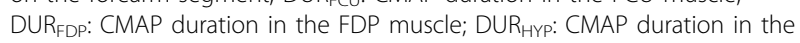
HYP area muscle after stimulus on the arm segment; DUR HYP-FA: CMAP duration in the HYP area muscles after stimulus on the forearm segment; SPSS: Statistical Package for the Social Sciences; $V_{F C U}$ : Ulnar nerve conduction velocity in the FCU muscle; $V_{\text {FDP }}$ : Ulnar nerve conduction velocity in the FDP muscle; $V_{\text {HYP: }}$ Ulnar nerve conduction velocity in the HYP area muscle after stimuli on the arm segment; $V_{\text {HYP-FA: Ulnar nerve }}$ conduction velocity in HYP area muscles after stimuli on the forearm segment; Cm: centimeter; m: meter; mV: milivolt; s: second; SD: Standard deviation; $\mu \mathrm{m}$ : micrometer; $\mu \mathrm{s}$ : microsecond; ${ }^{\circ} \mathrm{C}$ : degree Celcius

\section{Acknowledgements}

No acknowledgements.

\section{Author details}

${ }^{1}$ Pamukkale University, School of Medicine, Department of Neurology, Araştırma Hastanesi, Kınıklı-Denizli, Turkey. ${ }^{2}$ Devlet Hastanesi, Siirt, Turkey. ${ }^{3}$ Asker Hastanesi, Eskişehir, Turkey.

\section{Authors' contributions}

SG and MÇ helped to perform EMG studies and to collect data. ED helped to translate into English and revised manuscript. AO designed study, performed EMG studies, collected data and wrote the article. All authors read and approved the final manuscript.

\section{Authors' information}

All authors are specialists in neurology. Additionally, AO is a professor in neurology and ED is an assistant professor in neurology.

\section{Competing interests}

The authors declare that they have no competing interests.

Received: 12 May 2010 Accepted: 13 September 2010 Published: 13 September 2010

\section{References}

1. Cullheim S: Relations between cell body size, axon diameter and axon conduction velocity of cat sciatic $\alpha$-motoneurons stained with horseradish peroxidase. Neurosci Lett 1978, 8:17-20.

2. Buchthal F, Schmalbruch H: Motor unit of mammalian muscle. Physiol Rev 1980, 60:90-142.

3. McHanwell S, Biscoe TJ: The sizes of motoneurons supplying hindlimb muscles in the mouse. Proc R Soc Lond B Biol Sci 1981, 213:201-16.

4. Oğuzhanoğlu A, Erdoğan Ç, Tabak E, Cenikli U: Comparison of conduction velocities of nerve fibers to smaller and larger muscles in rats. Int J Neurosci 2010, 120:76-79.

5. Henneman $\mathrm{E}$, Olson $\mathrm{CB}$ : Relations between structure and function in the design of skeletal muscles. J Neurophysiol 1965, 28:581-598.

6. Gassel MM, Trojaborg W: Clinical and electrophysiological study of the pattern of conduction times in the distribution of the sciatic nerve. $J$ Neurol Neurosurg Psychiatry 1964, 27:351-7.

7. Felsenthal G, Brockman PS, Mondell DL, Hilton EB: Proximal forearm ulnar nerve conduction techniques. Arch Phys Med Rehabil 1986, 67:440-4.

8. Delagi EF, Perotto A: Anatomic Guide for Electromyographer Springfield, IL, Thomas, 21980

9. Fernand VS, Young JZ: The sizes of the nerve fibres of muscle nerves. Proc R Soc Lond B Biol Sci 1951, 139:38-58.

10. Devanandan MS, Ghosh S, Simoes EA: Myelinated fibers of the deep branch of the ulnar nerve at the wrist in bonnet monkeys (Macaca radiata) and some of its branches to the hand. Anat Rec 1980, 197:387-96.

11. Harding C, Halar E: Motor and sensory ulnar nerve conduction velocities: effect of elbow position. Arch Phys Med Rehabil 1983, 64:227-32.

12. Buschbacher RM: Ulnar nerve motor conduction to the abductor digiti minimi. Am J Phys Med Rehabil 1999, 78(6 Suppl):S9-14.

13. Johnsen B, Fuglsang-Frederiksen A, de Carvalho M, Labarre-Vila A, Nix W, Schofield I: Amplitude, area and duration of the compound muscle action potential change in different ways over the length of the ulnar nerve. Clin Neurophysiol 2006, 117:2085-92. 
14. Olney RK, Budingen HJ, Miller RG: The effect of temporal dispersion on compound action potential area in human peripheral nerve. Muscle Nerve 1987, 10:728-33.

15. Kimura J, Sakimura Y, Machida M, Fuchigami Y, Ishida T, Claus D, et al: Effect of desynchronized inputs on compound sensory and muscle action potentials. Muscle Nerve 1988, 11:694-702.

16. Trojaborg W: Motor nerve conduction velocities in normal subjects with particular reference to the conduction in proximal and distal segments of median and ulnar nerve. Electroencephalogr Clin Neurophysiol 1964, 17:314-21

17. Spiegel MH, Johnson EW: Conduction velocity in the proximal and distal segments of the motor fibers of the ulnar nerve of human beings. Arch Phys Med Rehabil 1962, 43:57-61.

18. Todnem K, Knudsen G, Riise T, Nyland H, Aarli JA: The non-linear relationship between nerve conduction velocity and skin temperature. J Neurol Neurosurg Psychiatry 1989, 52(4):497-501.

\section{Pre-publication history}

The pre-publication history for this paper can be accessed here: http://www.biomedcentral.com/1471-2377/10/81/prepub

doi:10.1186/1471-2377-10-81

Cite this article as: Oğuzhanoğlu et al:: Conduction in ulnar nerve bundles that innervate the proximal and distal muscles: a clinical trial. BMC Neurology 2010 10:81.

\section{Submit your next manuscript to BioMed Central and take full advantage of:}

- Convenient online submission

- Thorough peer review

- No space constraints or color figure charges

- Immediate publication on acceptance

- Inclusion in PubMed, CAS, Scopus and Google Scholar

- Research which is freely available for redistribution

Submit your manuscript at www.biomedcentral.com/submit 